\title{
Rearfoot and knee coupling over a prolonged run in runners with patellofemoral pain syndrome Irene S Davis*1 and Tracy A Dierks²
}

\author{
Address: ${ }^{1}$ University of Delaware, Newark, DE, USA and ${ }^{2}$ Indiana University, Indianapolis, IN, USA \\ Email: Irene S Davis* - mcclay@udel.edu \\ * Corresponding author
}

from Ist Congress of the International Foot \& Ankle Biomechanics (i-FAB) community

Bologna, Italy. 4-6 September 2008

Published: 26 September 2008

Journal of Foot and Ankle Research 2008, I(Suppl I):OI5 doi:I0.I I86/I757-I I46-I-SI-OI5

This abstract is available from: http://www.jfootankleres.com/content/I/SI/OI5

(c) 2008 Davis and Dierks; licensee BioMed Central Ltd.

\section{Introduction}

Patellofemoral pain syndrome (PFPS) is common in runners and is often related to excessive rearfoot eversion. Increased eversion is known to be associated with increased knee flexion, internal rotation, and reduced adduction [1]. Therefore, our purpose was to investigate coupling between the foot and knee in runners with PFPS. We hypothesized that the PFPS group would display larger coupling angles at baseline, due to relatively greater eversion. We also expected that these angles would increase to a greater degree in the PFPS group at the end of a prolonged run.

\section{Methods}

20 runners (18-40 yrs) with PFPS and 20 controls (CON) participated in a prolonged treadmill run averaging 30-45

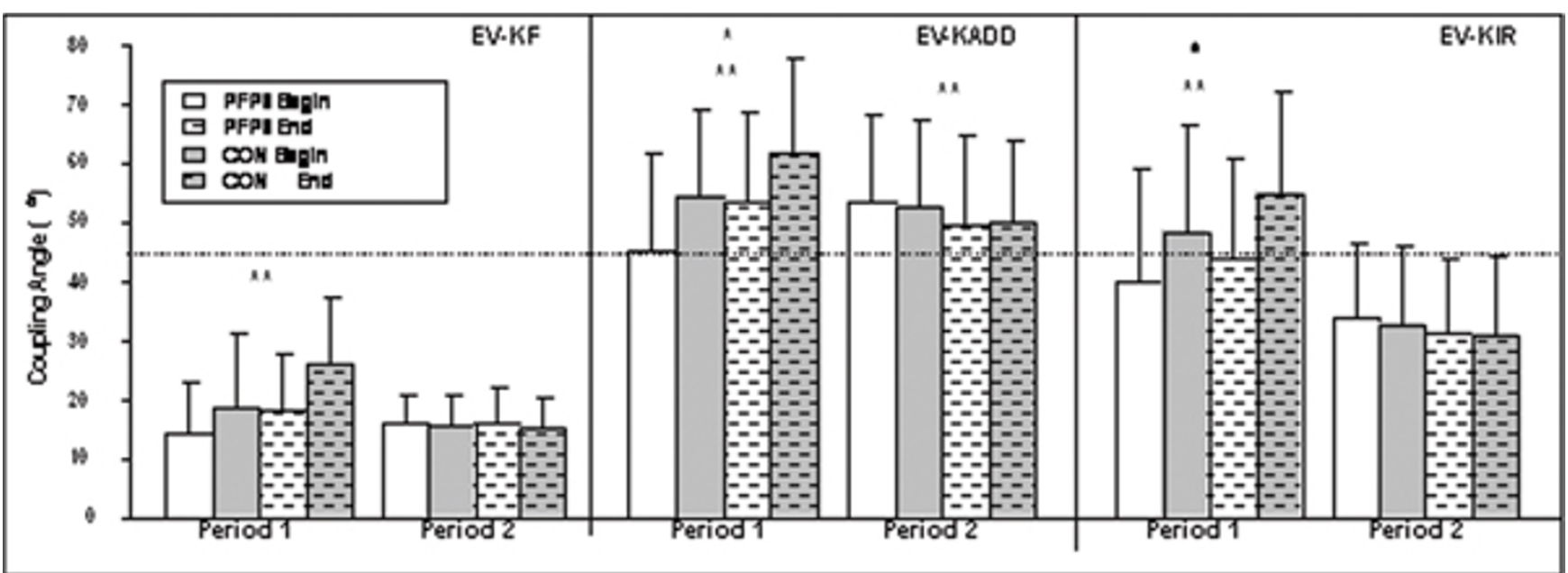

Figure I

Coupling angle group means during the 2 loading periods of stance at the beginning and end of the run. $*=$ Significant main effect for group in a period. $* *=$ Significant main effect for time in a period. Horizontal dashed line at $45^{\circ}$ indicates point of equal amounts of EV and knee excursion. 
min. The run was stopped when preset criteria of heart rate, perceived exertion or knee pain were reached. 3D kinematic data were collected at the beginning and end of the run. A vector coding method [2] was used to assess the following joint couplings: rearfoot eversion (EV) with knee flexion (KF), adduction (KADD), and internal rotation (KIR). Coupling angles of $45^{\circ}$ indicate equal joint excursions. Increasing values indicate more eversion, decreasing values indicate more knee motion. Values were averaged over the first half of stance: Period 1 (initial loading): footstrike to vertical impact peak (VIP) and Period 2 (maximal loading): VIP to vertical propulsive peak. A 2factor ANOVA (group by time) was used ( $p=0.05$ ).

\section{Results}

There were no group by time interactions. During period 1 , all coupling values were unexpectedly lower in the PFPS group, with EV-KADD and EV-KIR couplings being significantly lower (Figure 1). This relatively reduced eversion may have been a compensatory control strategy to minimize the abnormal knee mechanics that often accompany excessive eversion. This period of early loading is important, as the patella is not yet well seated into the femoral trochlea and is vulnerable to malalignment. By period 2, coupling angles were similar between the 2 groups for all relationships. As this is the period of maximal loading, the PFPS group may not have been able to maintain the relatively reduced EV seen in early loading. Over the course of the run, both groups increased their coupling angles in period 1 at the end of the prolonged run compared to the beginning. This may be an indication of fatigue, as EV has been shown to increase over the course of prolonged running [3].

\section{Conclusion}

Coupling angles are lower at initial loading in the PFPS group. During maximal loading, coupling is generally similar between groups. Finally, both groups increased their coupling angles over the course of the prolonged run.

\section{References}

I. McClay I, et al.: Clin Biom 1998, I 3: 195-203.

2. Sparrow A, et al.: J Mot Beh 1987, 19:1 15-129.

3. Derrick T, et al.: MSSE 2002, 34:998-1002.

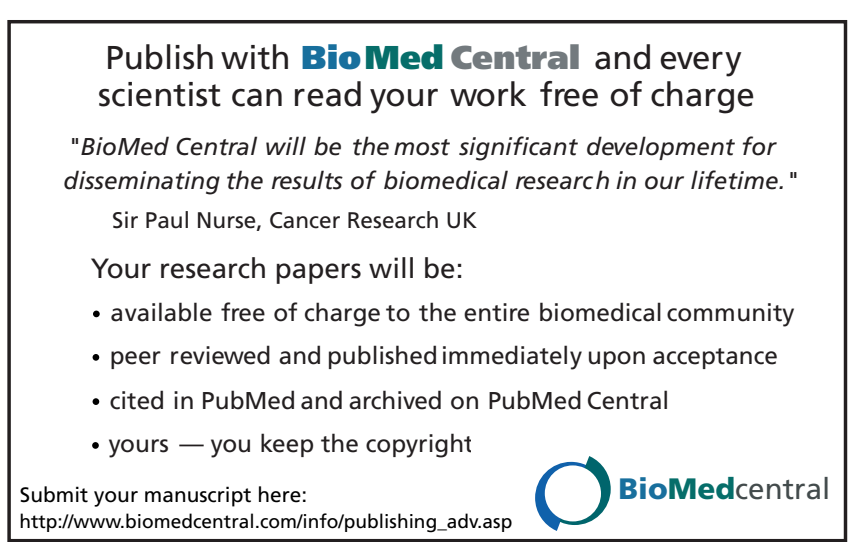

Page 2 of 2

(page number not for citation purposes) 